\title{
Olanzapine in Chemotherapy Induced Nausea and Vomiting
}

\author{
Syed Saad Hussain ${ }^{*}, 1$, Fasiha Abdul Rahman², Sarmad Iqbal ${ }^{3}$ \\ ${ }^{I}$ Department of Pharmacy, Iqra University, Karachi, Pakistan. \\ ${ }^{2}$ Department of Pharmacy, University of Karachi, Karachi, Pakistan. \\ ${ }^{3}$ Department of Pharmacy Practice, University of Karachi, Karachi, Pakistan.
}

\begin{abstract}
Objective: Patients diagnosed with cancer and undergoing chemotherapeutic regimen often anticipate many negative effects of the therapy. Chemotherapy induced nausea and vomiting is of their principal concerns which remains one of the most unpleasant, distressing and feared adverse effect. The management of chemotherapy induced nausea and vomiting has always been a challenge for high and moderate emetogenic chemotherapeutic agent. $\mathrm{D}_{2}$ receptor antagonists were the first agents to produce antiemetic effect. Then 5-HT 3 receptor antagonist and $\mathrm{NK}_{1}$ receptor antagonist were developed which proved to possess higher selective antiemetic effect compared to $\mathrm{D}_{2}$ receptor antagonist. This review includes the clinical advancements of an atypical antipsychotic agent olanzapine and its role in chemotherapy induced nausea and vomiting. Olanzapine is traditionally used to treat schizophrenia and manic disorder. But recent clinical studies have illustrated its additional therapeutic effect as an antiemetic agent. This advancement may prove to have beneficial effect in managing chemotherapy induced nausea and vomiting and providing a better quality of life to patient being treated with different chemotherapeutic agents.
\end{abstract}

Keywords: Anti-emetic, CINV, Chemotherapy, Anti-psychotic, Emetogenicity, Clinical Trial.

\section{INTRODUCTION}

The frequency and severity of adverse effects are the distinguishing characteristics between anticancer agents and other drugs at therapeutic doses [1]. In order to reduce these toxic effects dose reduction, use of alternate drugs or their analogues, growth factors and cytoprotective agents are used [2]. Toxic effects commonly observed with anticancer agents include nausea and vomiting, hair loss, stomatitis and leukopenia [3]. The probability of developing chemotherapy induced nausea and vomiting depends on several factors ranging from sex and age to anticancer agent administered to the patient [4].

Nausea and vomiting are regarded as the most unpleasant and distressing side effect of cancer chemotherapy. CINV may occur within hours of administration (acute), or after 24 hours and persist over several days. The development of ondansetron $\left(5-\mathrm{HT}_{3}\right.$ receptor antagonist) provided with better prophylaxis in of nausea and vomiting associated with moderate and highly emetogenic chemotherapeutic agent [5].

In this study we have reviewed an atypical antipsychotic agent i.e., olanzapine. It belongs to the antipsychotic category of therapeutic agents, but it also possesses the ability to effectively control nausea and emesis in all phases of CINV. Its antiemetic actions have been owed to its potential to antagonize various neurotransmitters and their receptor subtypes that have been implicated in CINV. The aim of this study is to

*Address correspondence to this author at the Department of Pharmacy, Iqra University, Karachi, Pakistan.

Email: saad.hussain96@outlook.com elaborate the anti-emetic effects of olanzapine and summarize related clinical trials in mitigating CINV. This study will prove a comprehensive document for the role of olanzapine in CINV.

\section{OLANZAPINE - AN ATYPICAL ANTIPSYCHOTIC}

Olanzapine, a thienobenzodiazepine compound, belongs to second genera or atypical group of antipsychotic drugs and possess a close structure with that of clozapine [6-8] and is therapeutically active in the treatment of neurological disorders including schizophrenia, mania, anorexia and bipolar disease $[6,7,9$, ]. Olanzapine being the second most non-traditional antipsychotic, it bears clinically insignificant potential for the undesirable adverse events for instance extrapyramidal symptoms (EPS), tardive dyskinesia, and neuroleptic malignant syndrome. These are otherwise commonly seen with the conventional agents and are a major concern with their utilization [6,7] and hence, olanzapine like other non-conventional drugs appears to have a better patient compliance with consequently improved efficacy $[6,7]$. Olanzapine has fulfilled the principal reason for its development, a drug with improved safety (comparatively lower propensity for agranulocytosis $[10,11]$ and yet comparable to its structurally similar counterpart, the atypical prototype "clozapine", in terms of effectiveness $[6,7,10]$.

The dosage forms available for olanzapine include both oral and parenteral (intramuscular) formulations, each approved with different dosage and indication. Oral olanzapine has a good absorption which is unaffected by food but subjected to an extensive (about $40 \%$ ) first-pass metabolization involving 
direct glucuronidation and cytochrome P450 - oxidative pathway.

\section{CHEMOTHERAPY INDUCED NAUSEA AND VOMIT- ING OR CINV}

CINV or chemotherapy - induced nausea and vomiting is a well-known undesirable event. It is perceived to be among the principal adverse events in relation to the cancer treatment $[12,13]$. It may produce several complications including aspiration pneumonia, dehydration state, electrolyte imbalance [13, 14], and malnourishment [12]. Consequently, it bears a great potential to affect quality of life of the person undergoing chemotherapy in adversative manner [12-15] and results in reduced therapeutic compliance and therapeutic effectiveness [14].
The extent of CINV and its risk may vary in accordance with chemotherapeutic drug (Emetogenicity [16]) and certain patient-specific characteristics $[13,17]$. It can be categorized as per its occurrence timing into acute-type (occurring within 24 hours post-chemotherapy $[13,14])$ and the delayed-type (occurring in 24 to 120 hours post - chemotherapy [13, 14]. Another type can be anticipatory emesis [18], occurring as conditioned response before the therapy in patients who had suffered this adverse event in the previous chemotherapeutic cycle.

\section{EMETOGENICITY}

The chemotherapeutic drugs are categorized in accordance with their potential for emesis induction (Table 1).

Table 1. Emetogenicity Categorization according to ESMO Guidelines.

\begin{tabular}{|l|l|}
\hline \multicolumn{1}{|c|}{ Category } & Definition \\
\hline Highly Emetogenic & $\begin{array}{l}\text { A cytotoxic agent is considered to possess high emetogenicity if the risk of including emesis } \\
\text { is above } 90 \% .\end{array}$ \\
\hline Moderately Emetogenic & $\begin{array}{l}\text { A cytotoxic agent is considered to be of moderate emetogenicity if the risk of including } \\
\text { emesis lies between } 30 \% \text { to } 90 \% .\end{array}$ \\
\hline Low Emetogenic & $\begin{array}{l}\text { A cytotoxic agent is considered to be of low emetogenicity if the risk of inducing emesis is } \\
\text { in range of } 10 \% \text { to } 30 \% .\end{array}$ \\
\hline Minimally Emetogenic & $\begin{array}{l}\text { A cytotoxic agent is said to has a minimal potential for emetogenicity if the risk of inducing } \\
\text { emesis is below } 10 \% .\end{array}$ \\
\hline
\end{tabular}

According to ESMO guideline, the agents included in highly emetogenic chemotherapy HEC category include anthracycline, carmustine, cisplatin, cyclophosphamide, dacarbazine, mechlorethamine and streptozotocin [19].

\section{PATHOPHYSIOLOGY OF CINV}

The pathophysiological process of CINV has principal involvement of gastrointestinal tract, central nervous system, and peripheral nervous system. The emetic reflex involves three regions, located in brainstem, including vomiting center (also called emetic pattern generator, EPG [18], Area Postrema (AP) and Nucleus Tractus Solitarius (NTS). The chemoreceptor trigger zone or CTZ is a collective term used for AP and NTS0.

Anatomically the vomiting center in medullary region is composed of effector nuclei and receptors. It is responsible for coordinating the vomiting reflex [18] via serving as final component of effector pathways in emesis activation for different afferent stimuli. The CTZ in the fourth ventricle (dorsal brainstem) serves as mean of afferent impulse to vomiting center. It is located outside the BBB or blood-brain barrier and consequently, in direct contact with blood and cerebrospinal fluid (CSF) [18]. Hence emetic triggers with blood or CSF origin have an easy accessibility to CTZ for emesis induction [18].

The pathophysiology of nausea and vomiting associated with cancer therapy may vary with every chemotherapy cycle [15] but overall activation and progression of CINV involve six receptors for the respective neurotransmitters involved [18]. The key neurotransmitters include serotonin (5-hydroxytryptamine or 5-HT), dopamine (D) and substance P [15]. The receptors implicated in $\mathrm{CINV}$ include $5-\mathrm{HT}_{2}, 5-\mathrm{HT}_{3}$, and $5-\mathrm{HT}_{4}$ for serotonin, $\mathrm{D}_{2}$ for dopamine, $\mathrm{NK}_{1}$ for substance $\mathrm{P}$, H1 for histamine and M1 for acetylcholine [18].

Chemotherapeutic agents induce nausea and vomiting via different ways. These include cellular damage at the level of intestinal tract, interacting with CTZ and coordination with the vomiting center [18]. However, the definite mechanism behind the emesis induction is still vague. The researches have so far concluded that chemotherapy-initiated emetic phenomenon occurs via central and peripheral pathway, each involving different neurotransmitters.

Peripheral mechanism principally involves serotonin and its $5 \mathrm{HT}_{3}$ receptors, located in intestine. Defense lines to put up with toxins include liver and stomach wall, with abundance of $\mathrm{D}_{2}$ and 5HT receptors in the enterochromaffin (EC) cells of the intestinal mucosa. Factors such as bowel distension, 
chemotherapeutic drug, radiotherapy etc., which results in mucosal damage, may greatly trigger the release of 5HT from these sites [18]. Serotonin once released interacts with $5 \mathrm{HT}_{3}$ receptors located on afferent fibers of splanchnic and vagus nerves in the intestinal wall. Input via these fibers is conveyed to chemoreceptor trigger zone (CTZ) to elicit an emetic response.

The central mechanism of emetic response has principal involvement of neurotransmitter substance $\mathrm{P}$ (and its $\mathrm{NK}_{1}$ receptor) and dopamine (and its predominant $\mathrm{D}_{2}$ receptors) [18]. Hence, 5HT seems to have a predominant involvement at initial (acute) stage of CINV but as it progresses towards delayed phase involvement of dopamine and substance-P also comes into play [15]. Moreover, afferent impulse via vagus nerve causing release of substance-P in the regions of area postrema and tractus solitarius, has also been implicated in the delayed phase and is mediated via $\mathrm{NK}_{1}$ receptor.

Some cytotoxic agents appear to have a direct or indirect interaction with the receptors found in the CTZ, consequently activating the vomiting center. Lastly, amygdala, a higher center in central nervous system of limbic forebrain serves as another powerful emetic stimulus and has a direct input to vomiting center [15].

\section{PHARMACOLOGICAL ACTION}

Olanzapine is a thio-benzodiazepine-class atypical antipsychotic with the ability to inhibit a variety of receptors, which explains its antiemetic properties. Target receptors for olanzapine include Dopaminergic receptors $\left(\mathrm{D}_{1}, \mathrm{D}_{2}, \mathrm{D}_{3}, \mathrm{D}_{4}\right)$, serotonergic $\left(5-\mathrm{HT}_{2 \mathrm{~A}}, 5-\mathrm{HT}_{2 \mathrm{C}}, 5-\mathrm{HT}_{3}, 5-\mathrm{HT}_{6}\right)$, muscarinic $(\mathrm{m} 1, \mathrm{~m} 2, \mathrm{~m} 3, \mathrm{~m} 4)$, adrenergic $(\alpha 1)$ and histaminergic $(\mathrm{H} 1)$ [20]. The benefit of olanzapine over conventional antiemetic regimens used for CINV is its ability target multiple key receptors with one medication. The convenience of once or twice daily administration and the use of a single agent will boost patient compliance in situations where taking drugs is difficult due to nausea and vomiting [21].

Oral drug absorption of olanzapine is $85 \%$, about $40 \%$ is inactivated by first-pass hepatic metabolism, resulting in a $60 \%$ oral bioavailability of the total dose absorbed. Olanzapine has a mean half-life of 33 hours in healthy people (range 21-54 hours). Peak plasma concentrations are reached within 6 hours of administration. 93\% drug is bound to plasma proteins, mostly albumin $(90 \%)$ and a1-acid glycoprotein $(77 \%)[22]$.

Olanzapine dosing is not affected by renal impairment. Urinary concentrations of olanzapine metabolite were notably increased in patients with cirrhosis while no statistical difference is observed between healthy and hepatic impaired patients receiving single dose olanzapine [23].

\section{MANAGEMENT OF CINV}

The nausea and emesis associated with chemotherapy can be managed via prophylactically using antiemetic drugs [12]. The main goal of using antiemetic is to completely prevent CINV in most of the cases of chemotherapy including those involving cytotoxic agents with high emetogenic potential.

Since the pathophysiological mechanisms of CINV have implication of various receptor types, [12] drugs with action on different receptors, belonging to different categories have been recommended for its control [15]. These drugs include $\mathrm{D}_{2}$ (dopamine) receptor antagonists, 5- $\mathrm{HT}_{3}$ (serotonin type-three) receptor antagonists, glucocorticoids, and substance-P (neurokinin-1) receptor antagonist [15]. Of these, the latter three classes of drugs have provided a great deal of control over CINV [16], demonstrating very high therapeutic efficacy for its management. Hence, most of the international guidelines pertaining to the management of CINV highly recommend 5- $\mathrm{HT}_{3}$ antagonists, $\mathrm{NK}_{1}$ (substance-P) antagonist and dexamethasone (glucocorticoid) to be used in combination [16].

These agents have been widely evaluated via several clinical trials where the primary focus has been the intravenous chemotherapy with either moderate or high emetogenic potential. Given the fact that all antiemetic protocols have not been evaluated against each chemotherapy protocol, results have been extrapolated rationally to chemotherapy protocols with similar emetogenic potential.

The recommended antiemetic combination has evidently provided an extensive control in reducing the occurrence of cancer treatment induced nausea and vomiting $[12,16]$. The efficacy is more evident in preventing acute type of CINV; hence the delayed and refractory types [15] of CINV still remain unresolved issues. The exact mechanism by which these antiemetic drugs prevent one type of CINV while failing to do so with the other types remains vague [12]. Conversely, this can be explained via pathophysiological understanding of CINV since involvement of other subtypes of dopamine and 5HT receptors has also been suggested [15]. The refractory type of nausea and emesis in the cases of chemotherapy with high emetogenic capacity has an estimated incidence of $20 \%$ to $40 \%$, even with the use of available antiemetic protocols [17]. Hence, CINV still prevails despite the compliance with recommended antiemetics drugs [15].

\section{OLANZAPINE AS AN ANTIEMETIC}

Olanzapine possess significant antiemetic effects as reported in various studies. The data from these studies and various clinical trials [17] has provided supporting evidence for its 
antiemetic efficacy in the treatment as well as prevention of nausea and emesis induced by chemotherapy, including both types: acute and delayed [15]. Additionally, it has been found to effectively manage breakthrough and refractory CINV [17].

The mechanism behind the clinical effectiveness of olanzapine in CINV is primarily based on its ability to block multiple neurotransmitters. The neurotransmitters blocked by olanzapine include 5-HT (serotonin), dopamine (D), histamine $(\mathrm{H})$, catecholamines and acetylcholine (Ach) [15]. Pertaining to the receptors of these neurotransmitters, olanzapine possess strong antagonism at $5 \mathrm{HT}_{2 \mathrm{~A}}, 5 \mathrm{HT}_{2 \mathrm{C}}, \mathrm{D}_{1}, \mathrm{D}_{2}, \mathrm{D}_{3}, \mathrm{D}_{4}, \alpha 1$ (adrenergic for catecholamine), and $\mathrm{H} 1$ receptor. It possess moderate affinity for muscarinic specifically $\mathrm{M} 1$ and $5 \mathrm{HT}_{3}$ receptors, and weak affinity for $\beta$ (adrenergic), benzodiazepine and $\mathrm{GABA}_{\mathrm{A}}$ receptors.

Different studies have been conducted comparing the effectiveness of olanzapine with the antiemetic agents that are being used and recommended by clinical guidelines in managing CINV.

A randomized clinical trial showed that olanzapine when administered for controlling breakthrough nausea and emesis in patients on chemotherapy; it prevented CINV in $68 \%$ of patients. In comparison, breakthrough CINV was found to be controlled in $23 \%$ of patients who were not administered olanzapine but metoclopramide [17]. Olanzapine was found to possess antiemetic efficacy equal to aprepitant, an $\mathrm{NK}_{1}$ receptor blocker, in the prevention of delayed type of CINV. However, the antiemetic efficacy was found to be higher than aprepitant in the prophylaxis of acute phase. An additional benefit of using olanzapine in place of NK1 receptor blocker can be its reduced cost, hence besides providing a better therapeutic control lower cost can also be a reason to increase patient compliance [14].

Furthermore, in a trial olanzapine when administered concomitantly with glucocorticoid and a $5 \mathrm{HT}_{3}$ receptor blocker (azasetron, granisetron or palonosetron), it showed effective prophylaxis of nausea and emesis induced by chemotherapeutic agents of high and moderate emetogenic potential. This combination was found to be effective for both phases of CINV, the acute and delayed [15].

A phase-3, randomized, placebo-controlled study compared the most commonly recommended antiemetic protocol (combination of a $5-\mathrm{HT}_{3}$ antagonists, an $\mathrm{NK}_{1}$ antagonist and dexamethasone) against the same regimen including olanzapine as the fourth drug in this combination. The patients included in the trial were being administered chemotherapy of high emetogenic potential and had no past experience of chemotherapy. In accordance with the results addition of olanzapine provided a more effective control in all phases of CINV, rendering it to be an effective antiemetic in managing nausea and emesis induced by cytotoxic agents [16].

Various trials assessing olanzapine pertaining to CINV have reported drowsiness to be the adverse event commonly experienced $[15,16]$. This effect appeared initially and subsided as the therapy was continued [16]. Other adverse events seen were edema and dry mouth but these along with drowsiness were of tolerable intensity. These were found to be resolved once olanzapine therapy was completed [15]. It has been suggested to administer oral olanzapine in a lower dose of $5 \mathrm{mg}$ since it has displayed efficacy equal to the dose of 10 $\mathrm{mg}$, in control of nausea and emesis in all CINV phases but has a lower potential for adverse events $[14,16]$.

The efficacy and safety study conducted by [24] including 13 children receiving moderate and high emetogenic chemotherapy regimen, complete response was achieved in $90.0 \%$ of the patients receiving olanzapine for HEC while complete response of $60 \%$ was observed in patients receiving MEC. The most common adverse effect observed in this study was somnolence (19.5\%), hyperglycemia (14.6\%), fatigue (2.4\%) and disturbed sleep (2.4\%).

A meta-analysis conducted by [25] provided class 1 evidence that for CINV protection, $10 \mathrm{mg}$ olanzapine in combination with palonosetron and dexamethasone is effective and well tolerated. The study also concluded that complete response was significantly higher in proportion of patients receiving olanzapine than control group.

A study of effectiveness of antiemetic regimens for highly emetogenic CINV compared different antiemetic regimens. Through direct and indirect meta-analysis, it was found that olanzapine containing regimens $\left(\mathrm{OLZ}+5-\mathrm{HT}_{3}+\mathrm{DEX}\right.$, OLZ $\left.+\mathrm{PAL}+\mathrm{DEX} \& \mathrm{OLZ}+\mathrm{NK}_{1}+5 \mathrm{HT}_{3}+\mathrm{DEX}\right)$ were the most effective compared to the same regimens excluding olanzapine with an overall complete response observed between 0.81 -0.99 on the SUCRA ranking scale. It also concluded that the substitution of olanzapine for an $\mathrm{NK}_{-1}$ receptor antagonist may be the most cost effective strategy for managing CINV [26].

In a systemic review, meta-analysis, and fragility assessment report of more than 25 studies with $4200+$ patients including one study on children, olanzapine was found to be statistically and clinically superior to other antiemetic regimens in the treatment of CINV breakthrough. Furthermore, Olanzapine is found to be statistically and clinically superior in seven of nine efficacy endpoints in the prophylaxis setting in studies reporting on HEC [27].

In a randomized, double blind, placebo-controlled study, 
safety, and efficacy of olanzapine on its effect on frequency of CINV and improved quality of life (QOL) in patients receiving palonosetron and dexamethasone as prophylaxis treatment for CINV. The study concluded that the combination of olanzapine, palonosetron, and dexamethasone substantially improved QOL and vomiting control in previously untreated MEC patients, but the effectiveness was limited to a decrease in the frequency of CINV [28].

On the grounds of various studies and the supporting evidence, $(\mathrm{NCCN})$ the National Comprehensive Cancer Network included olanzapine in its guidelines of 2016. In accordance with this, combination of olanzapine with dexamethasone and palonosetron has been recommended as an alternate protocol for the CINV prophylaxis. Other recommendations for managing breakthrough CINV include consideration of an olanzapine containing combination for next cycles of chemotherapy in patients who were on regimen containing $\mathrm{NK}_{1}$ blockers and adding a drug belonging to unused antiemetic group, for instance, olanzapine [17].

\section{CONCLUSION}

In these studies, it has been compared with various antiemetic agents and recommended antiemetic regimens that are being used for managing the nausea and emesis adverse effect of chemotherapeutic agents. Its addition to standard antiemetic protocol has been found superior in managing nausea and emesis in different phases of CINV. Furthermore, olanzapine is economical having no associated serious adverse events. These along with its ability to provide effective control of CINV have rendered it as a potentially effective antiemetic agent to be added in the regimens recommended by international guidelines pertaining to the management of CINV.

\section{AUTHORS' CONTRIBUTION}

Fasiha Abdul Rahman: Review design.

Fasiha Abdul Rahman, Syed Saad Hussain: Data collection.

Fasiha Abdul Rahman Syed Saad Hussain, Sarmad Iqbal: Draft manuscript preparation.

\section{CONFLICT OF INTEREST}

Declared none.

\section{ACKNOWLEDGEMENTS}

Declared none.

\section{REFERENCES}

[1] Remesh A. Toxicities of anticancer drugs and its management. Int J Basic Clin Pharmacol 2012; 1(1): 1-12. DOI: 10.5455/2319-2003.ijbcp000812

[2] Ritter JM, Flower RJ, Henderson G, Loke YK, Rang HP,
MacEwan D. Rang and Dale's Pharmacology. Netherlands: Elsevier 2019; pp: 1-808.

[3] Plenderleith IH. Treating the treatment: toxicity of cancer chemotherapy. Can Fam Physician 1990; 36: 1827-30.

[4] Hesketh PJ. Chemotherapy-induced nausea and vomiting. New Engl J Med 2008; 358(23): 2482-94. DOI: 10.1056/NEJMra0706547

[5] Cohen L, De Moor CA, Eisenberg P, Ming EE, Hu H. Chemotherapy-induced nausea and vomiting - incidence and impact on patient quality of life at community oncology settings. Supportive Care Cancer 2007; 15(5): 497-503. DOI: 10.1007/s00520-006-0173-Z

[6] Mauri MC, Paletta S, Maffini M, et al. Clinical pharmacology of atypical antipsychotics: An update. EXCLI J 2014; 13: 1163-91.

[7] Waller DG, Sampson T. Medical Pharmacology and Therapeutics E-Book: Netherlands: Elsevier Health Sciences 2013.

[8] Saudemont G, Prod'Homme C, Da Silva A, Villet SM, Reich NP. The use of olanzapine as an antiemetic in palliative medicine: A systematic review of the literature. BMC Palliative Care 2020; 19: 56. DOI: 10.1186/s12904-020-00559-4

[9] Attia E, Steinglass JE, Walsh BT, et al. Olanzapine versus placebo in adult outpatients with anorexia nervosa: A randomized clinical trial. Am J Psychiatry 2019; 176(6): 449-56. DOI: 10.1176/appi.ajp.2018.18101125

[10] Bymaster FP, Rasmussen $\mathrm{K}$, Calligaro DO, et al. In vitro and in vivo biochemistry of olanzapine: A novel, atypical antipsychotic drug. Clin Psychiatry 1997; 58(Suppl 10): 28-36.

[11] Meltzer HY, Fibiger HC. Olanzapine: A new typical antipsychotic drug. Neuropsychopharmacology 1996; 14(2): 83-5. DOI: 10.1016/0893-133X(95)00197-L

[12] Mizukami N, Yamauchi M, Koike K, et al. Olanzapine for the prevention of chemotherapy-induced nausea and vomiting in patients receiving highly or moderately emetogenic chemotherapy: A randomized, double-blind, placebo-controlled study. J Pain Symptom Manage 2014; 47(3): 542-50. DOI: 10.1016/j.jpainsymman.2013.05.003

[13] Navari RM. Management of Chemotherapy-Induced Nausea and Vomiting: New Agents and New Uses of Current Agents: USA: Springer International Publishing 2016. DOI: 10.1007/978-3-319-27016-6

[14] Yang T, Liu Q, Lu M, Ma L, Zhou Y, Cui Y. Efficacy of olanzapine for the prophylaxis of chemotherapy-induced nausea and vomiting: A meta-analysis. Br J Clin Pharmacol 2017; 83(7): 1369-79. DOI: 10.1111/bcp.13242

[15] Vig S, Seibert L, Green MR. Olanzapine is effective for refractory chemotherapy-induced nausea and vomiting irrespective of chemotherapy emetogenicity. J Cancer Res Clin Oncol 
2014; 140(1): 77-82. DOI: 10.1007/s00432-013-1540-z

[16] Navari RM, Qin R, Ruddy KJ, et al. Olanzapine for the prevention of chemotherapy-induced nausea and vomiting. New Engl J Med 2016; 375(2): 134-42. DOI: 10.1056/NEJMoa1515725

[17] Laub M, Kator S. Olanzapine for the prevention and treatment of chemotherapy-induced nausea and vomiting. J Nurse Pract 2017; 13(4): 309-10. DOI: 10.1016/j.nurpra.2016.12.022

[18] Antonarakis ES, Hain RD. Nausea and vomiting associated with cancer chemotherapy: Drug management in theory and in practice. Arch Dis Childhood 2004; 89(9): 877-80. DOI: 10.1136/adc.2003.037341

[19] Roila F, Molassiotis A, Herrstedt J, et al. 2016 MASCC and ESMO guideline update for the prevention of chemotherapyand radiotherapy-induced nausea and vomiting and of nausea and vomiting in advanced cancer patients. Ann Oncol 2016; 27(Supple 5): v119-v33. DOI: 10.1093/annonc/mdw270

[20] Bradfford MV, Glode A. Olanzapine: An antiemetic option for chemotherapy-induced nausea and vomiting. J Adv Pract Oncol 2014; 5(1): 24-9. DOI: 10.6004/jadpro.2014.5.1.8

[21] Srivastava M, Brito-Dellan N, Davis MP, Leach M, Lagman R. Olanzapine as an antiemetic in refractory nausea and vomiting in advanced cancer. J Pain Symptom Manage 2003; 25(6): 578-82. DOI: 10.1016/S0885-3924(03)00143-X

[22] Callaghan JT, Bergstrom RF, Ptak LR, Beasley CM. Olanzapine: Pharmacokinetic and Pharmacodynamic Profile. Clin Pharmacokinet 2012; 37(3): 177-93. DOI: 10.2165/00003088-199937030-00001
[23] Mauri MC, Paletta S, Di Pace C, et al. Clinical pharmacokinetics of atypical antipsychotics: An update. Clin Pharmacokinet 2018; 57(12): 1493-528. DOI: 10.1007/s40262-018-0664-3

[24] Lee SR, Kim SM, Oh MY, Lee JM. Efficacy of olanzapine for high and moderate emetogenic chemotherapy in children. Children 2020; 7(9): 140. DOI: 10.3390/children7090140

[25] Eshak Ibrahim Bahbah EI, Abdalla AR, Abdelshafy K, et al. Should olanzapine be advocated over conventional anti-emetics for the prevention of chemotherapy-induced nausea and vomiting? an updated meta-analysis of randomized control trials. Curr Enzyme Inhibit 2019; 15(2): 80-90. DOI: $10.2174 / 1573408015666190620165507$

[26] Yokoe T, Hayashida T, Nagayama A, et al. Effectiveness of antiemetic regimens for highly emetogenic chemotherapy-induced nausea and vomiting: A systematic review and network meta-analysis. Oncologist 2019; 24(6): e347-e57. DOI: 10.1634/theoncologist.2018-0140

[27] Chow R, Herrstedt J, Aapro M, et al. Olanzapine for the prophylaxis and rescue of chemotherapy-induced nausea and vomiting: A systematic review, meta-analysis, cumulative meta-analysis and fragility assessment of the literature. Support Care Cancer 2021; 29(7): 3439-59. DOI: 10.1007/s00520-020-05935-7

[28] Jeon S-Y, Han HS, Bae WK, et al. A Randomized, double-blind, placebo-controlled study of the safety and efficacy of olanzapine for the prevention of chemotherapy-induced nausea and vomiting in patients receiving moderately emetogenic chemotherapy: Results of the Korean South West Oncology Group (KSWOG) study. Cancer Res Treat 2019, 51(1): 90-7. DOI: $10.4143 /$ crt.2017.577 